\title{
Integrating clinical and genetic approaches in the diagnosis of $46, X Y$ disorders of sex development
}

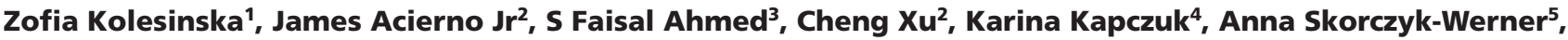 \\ Hanna Mikos ${ }^{1}$, Aleksandra Rojek ${ }^{1}$, Andreas Massouras ${ }^{6}$, Maciej R Krawczynski ${ }^{5}$, Nelly Pitteloud ${ }^{2}$ and \\ Marek Niedziela'
}

${ }^{1}$ Department of Pediatric Endocrinology and Rheumatology, Poznan University of Medical Sciences, Poznan, Poland ${ }^{2}$ Endocrinology, Diabetology \& Metabolism Service, Lausanne University Hospital, Lausanne, Switzerland

${ }^{3}$ Developmental Endocrinology Research Group, School of Medicine, Dentistry \& Nursing, University of Glasgow, Glasgow, UK ${ }^{4}$ Division of Gynecology, Department of Perinatology and Gynecology, Poznan University of Medical Sciences, Poznan, Poland ${ }^{5}$ Department of Medical Genetics, Poznan University of Medical Sciences, Poznan, Poland

${ }^{6}$ Saphetor, SA, Lausanne, Switzerland

Correspondence should be addressed to M Niedziela: marek.niedziela@ump.edu.pl

\begin{abstract}
$46, \mathrm{XY}$ differences and/or disorders of sex development (DSD) are clinically and genetically heterogeneous conditions. Although complete androgen insensitivity syndrome has a strong genotype-phenotype correlation, the other types of 46,XY DSD are less well defined, and thus, the precise diagnosis is challenging. This study focused on comparing the relationship between clinical assessment and genetic findings in a cohort of well-phenotyped patients with 46,XY DSD. The study was an analysis of clinical investigations followed by genetic testing performed on 35 patients presenting to a single center. The clinical assessment included external masculinization score (EMS), endocrine profiling and radiological evaluation. Array-comparative genomic hybridization (array-CGH) and sequencing of DSD-related genes were performed. Using an integrated approach, reaching the definitive diagnosis was possible in 12 children. The correlation between clinical and genetic findings was higher in patients with a more severe phenotype (median EMS 2.5 vs $6 ; P=0.04$ ). However, in 13 children, at least one variant of uncertain significance was identified, and most times this variant did not correspond to the original clinical diagnosis. In three patients, the genetic studies guided further clinical assessment which resulted in a reclassification of initial clinical diagnosis. Furthermore, we identified eight patients harboring variants in more than one DSD genes, which was not seen in controls $(2.5 \% ; P=0.0003)$. In summary, taking into account potential challenges in reaching the definitive diagnosis in $46, X Y$ DSD, only integrated approach seems to be the best routine practice.
\end{abstract}

\section{Key Words}

- array-comparative genomic hybridization

- differences and/or disorders of sex development

- massive parallel/next generation sequencing

- oligogenicity

- 46,XY DSD

\section{Introduction}

Differences and/or disorders of sex development (DSD) represent rare congenital conditions in which gonadal, chromosomal or anatomical sex is atypical. Due to its heterogeneous clinical presentation and genetic architecture, achieving a definitive diagnosis is challenging, especially when associated with 46,XY karyotype (1). Indeed, with the exception of several conditions with welldefined phenotypes that point to a specific underlying
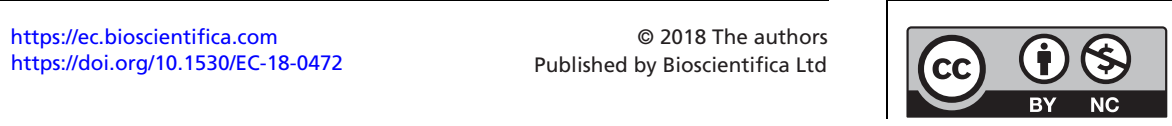

This work is licensed under a Creative Commons Attribution-NonCommercial 4.0 International License. 
genetic cause, i.e. complete androgen insensitivity (CAIS) (2), in the majority of cases neither the clinical presentation nor the phenotype-genotype correlation is indicative enough to predict a precise diagnosis.

The observed phenotypic heterogeneity is partially explained by complex mechanisms that control male sex development, and when disturbed may result in disorder of gonadal development (DGD), disorder of androgen synthesis (DAS) or disorder of androgen action (DAA) (1). Similar to the diverse pathophysiology, the genetic background varies with more than 40 genes having been implicated in its pathogenicity and associated with different modes of inheritance including autosomal dominant, autosomal recessive, $\mathrm{X}$-linked and de novo (1). Moreover, some DSD conditions may result from copy number variations (CNVs), especially in cases with gonadal dysgenesis or in the presence of associated malformations $(3,4,5)$. In addition, recent findings (6, $7,8)$ indicate oligogenicity, defined as mutation in more than one gene, as a possible mode of inheritance in DSD.

Therefore, in the light of phenotypic variability and the multiple genes associated with the phenotype, the introduction of next-generation sequencing (NGS) methods into the field of DSD significantly improved the diagnostic yield $(6,9)$. While whole-exome and/or genome sequencing (WES/WGS) are still reserved for the research environment, targeted gene panels are becoming widely used in the diagnostic algorithms of 46,XY DSD on a routine basis (10). However, as their utility was mostly proven in research genetic centers, there are still missing data regarding its application in a clinical setting, where reporting the genetic result to the patient and their family might be complicated by low sensitivity and/or specificity of endocrine tests that are unable to fully define the phenotype (11), potentially inconclusive results with the identification of a variant of uncertain significance or the increasingly stronger argument for oligogenicity as a possible mode of inheritance. This study aimed to compare the relationship between the clinical diagnosis (based on physical examination, biochemical and radiological assessment) to the result of genetic assessment using array-comparative genomic hybridization (arrayCGH) and sequencing 37 genes known to underlie 46,XY DSD in a cohort of 35 patients.

\section{Material and methods}

\section{Study group}

The study group initially consisted of 36 patients (34 probands) diagnosed with 46,XY DSD who were referred to a multidisciplinary clinic at a single pediatric endocrinology department for further investigations. Patients presented with either atypical genitalia in infants and children or atypical sexual development in adolescents. The appearance of external genitalia was described using the external masculinization score (EMS) (12), and consistent with UK guidance (13), only those with an EMS less than 11 were included. During the routine genetic testing prior to this study, one patient with complete gonadal dysgenesis was found to harbor a causative mutation in the $S R Y$ gene, and therefore, was excluded from the present study. Among 35 patients, 21 were raised as boys, while the remaining 14 were raised as girls. The cohort was classified according to above mentioned guidelines (13), with an exception of a diagnosis of a non-specific disorder of undermasculinization (NSDUM) assigned to those boys with normal testicular function before the analysis of the $A R$ gene (14), and a diagnosis of syndromic 46,XY DSD assigned to those with multiple ( $>1)$ associated conditions. The definition of small for gestational age (SGA) was a birth weight less than the tenth centile.

The study was approved by the Poznan University of Medical Sciences Medical Ethical Committee (Registration number 505/13) and informed consent was obtained from all participants.

\section{Endocrine investigations}

Standard hormonal measurements were performed using commercial kits. Luteinizing hormone (LH), follicle-stimulating hormone (FSH) and estradiol (E2) were assessed using chemiluminescent microparticle immunoassay (CMIA) by Architect (Abbott Laboratories) and dihydrotestosterone (DHT) by DHT-optimized ELISA (DRG Instruments GmbH, Germany). Androstenedione (A) and testosterone were measured by radioimmunoassays: Active Androstenedione RIA (Beckman Coulter, Immunotech) and TESTO-RIA-CT (DIAsource ImmunoAssays S.A., Belgium), respectively. Anti-Müllerian hormone $(\mathrm{AMH})$ and inhibin $\mathrm{B}$ were evaluated by ELx800 Absorbance Microplate Reader (BioTek Instruments) using Gen II ELISA assay (Beckman Coulter, Diagnostic Systems Laboratories, Inc.). The protocol for the hCG test was based on the administration of 1000 IU of hCG (Pregnyl) intramuscularly for three consecutive days with blood drawn immediately prior to the test on day one and $24 \mathrm{~h}$ after the last injection. An adequate increase of testosterone after hCG was considered to be sufficient if testosterone was over 3.5 (nmol/L) (15). Testosterone/DHT ratio was recognized

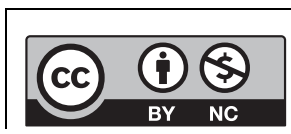

This work is licensed under a Creative Commons Attribution-NonCommercial 4.0 International License. 
as elevated if over 25 (16), while testosterone/A ratio was considered as decreased if below 0.8 (17). The GnRH test was conducting using an intravenous administration of $2.5 \mu \mathrm{g}$ of gonadorelinum (Relefact LH-RH) per kilogram (max. $100 \mu \mathrm{g}$ ) with a blood drawn prior to the test and at $30 \mathrm{~min}$ and at $60 \mathrm{~min}$ after injection. The assessment of internal genitalia (presence or absence of uterus, localization of undescended gonads) was performed using transabdominal ultrasound examination.

\section{Genetic investigations}

After a thorough literature and database search (OMIM, HGMD and ClinVar), a list of 37 genes implicated in $46, X Y$ DSD and included in the TruSight One sequencing kit (Illumina) were selected for evaluation (Table 1). If the gene was only reported in a single human case or family, then strong supporting in vitro or in vivo data must also be present for the gene to be included (CBX2, FGFR2, WWOX, and TSPY1) as stated in 'Guidelines for investigating causality of sequence variants in human disease' (18). Following other studies on the genetic etiology of 46 ,XY DSD $(6,7)$, we also evaluated a list of 21 genes implicated in congenital hypogonadotropic hypogonadism $(\mathrm{CHH})$ (Supplementary Table 1, see section on supplementary data given at the end of this article), a condition that shares some clinical presentations with DSD (i.e. cryptorchidism or micropenis) to further study their contribution. Reference transcript numbers for all genes evaluated are noted in Supplementary Table 2.

Blood samples were collected and frozen at $-20^{\circ} \mathrm{C}$ until the analysis, and molecular studies were performed on genomic DNA isolated from peripheral blood leukocytes using FlexiGene DNA Kit (QIAGEN).

Array-CGH was performed using a custom whole genome $180 \mathrm{~K}$ oligonucleotide array (Sure Print G3 Human 4x180K) manufactured by Agilent Technologies. The median probe spacing for the arrays was $11 \mathrm{~kb}$. Digestion, labeling and hybridization were completed according to manufacturer's protocols. Scanning and image analysis were performed as per oligonucleotide array-CGH protocol (Agilent, version 4.0). Microarrays were scanned using the Agilent Scanner. Agilent CytoGenomics Analytics software (v4.0.3.12) was used to visualize, detect and analyze the CNVs from microarray profiles using Tiff images from data. Identified CNVs were cross-checked with data gathered from the following databases: DGV (Database of Genomic Variants) (http:// dgv.tcag.ca/dgv/app/home), ISCA (International Standard for Cytogenomic Arrays) (http://dbsearch.clinicalgenome. org/search), NCBI (National Center for Biotechnology Information) (https://ncbi.nlm.nih.gov), as well as a local database of CNV.

For sequencing of the 37 DSD-related genes, patients' DNA samples were normalized to $5 \mathrm{ng} / \mu \mathrm{L}$ in $10 \mu \mathrm{L}$ of Tris- $\mathrm{HCl} 10 \mathrm{mM}$ pH 8.5 (total DNA mass $50 \mathrm{ng}$ ). Libraries were constructed for NGS using the TruSight One Sequencing Panel Kit (Illumina, Inc.) according to the manufacturer's protocol, and 150 basepair paired-end reads were generated on the MiSeq instrument (Illumina). Sample runs were validated as successful if the sequencing run yield was $>7.5 \mathrm{~Gb}$, had $>96 \%$ of reads aligning to the human genome and had an overall $30 \times$ coverage of $>95 \%$. Each run included 3 samples. Only variants with a read depth coverage of $>10 \times$ and a quality score of $>95$ passed quality control. Given these stringent parameters for genotype calls, Sanger sequencing was not required for further confirmation (19).

The raw data (fastq files) from the MiSeq sequencing of the TruSight One libraries were uploaded to Saphetor (www.saphetor.com, Lausanne, Switzerland); however, a mask was applied to the data such that only the 37 DSDrelated genes were analyzed. This analysis consisted of read alignments, basepair calling and annotation with HGVS (Human Genome Variation Society) nomenclature, function and conservation. Splice-junction $( \pm 6$ base pairs) and non-synonymous variants with a minor allele frequency (MAF) <1\% in Genome Aggregation Database (GnomAd) were evaluated. Of note, a missense variant was considered to be damaging if it was predicted to be damaging by one of the two in silico predictive algorithms, SIFT and/or MutationTaster. The variants were classified into five categories according to the American College of Medical Genetics (ACMG) guidelines (20) using the InterVar program (http://wintervar.wglab.org/): pathogenic, likely pathogenic and variants of uncertain significance (VUS), likely benign, and benign. Variants classified as benign or likely benign were not included in this study. Additionally, as previously described (6), any expansions in the first exon of the $A R$ gene of CAG over 27 repeats (21) or CGG over 24 repeats (22) were classified as VUS, as their contribution into DSD phenotype is still uncertain. A definitive (genetically confirmed) diagnosis was considered to be achieved when pathogenic or likely pathogenic variants were identified.

Oligogenicity of the DSD cohort was assessed against the 'Cohorte Lausannoise' (CoLaus) control population, consisting of 405 participants of mixed European origin, phenotyped as described by Firmann et al., in whom whole exome sequencing was performed (23).

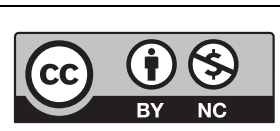
This work is licensed under a Creative Commons
Attribution-NonCommercial 4.0 International License. 
Table 1 46,XY DSD-related genes (37 genes).

\begin{tabular}{|c|c|c|c|c|}
\hline Gene & Phenotype & Inheritance & Location & OMIM \\
\hline \multicolumn{5}{|c|}{ Disorder of gonadal development (DGD) } \\
\hline$A R X$ & X-linked lissencephaly with abnormal genitalia & $X L$ & Xp21.3 & 300382 \\
\hline ATRX & $\begin{array}{l}\text { Alpha thalassemia X-linked intellectual } \\
\text { disability syndrome }\end{array}$ & $X L$ & $\mathrm{Xq} 21.1$ & 300032 \\
\hline$C B \times 2$ & CGD & $A R$ & $17 q 25.3$ & 602770 \\
\hline$D H H$ & CGD or PGD with or without polyneuropathy & $A R$ & $12 q 13.12$ & 605423 \\
\hline$D M R T 1$ & $\begin{array}{l}\text { CGD or PGD with dysmorphic features ( } 9 p \\
\text { monosomy syndrome) }\end{array}$ & $A D$, deletion & $9 p 24.3$ & 602424 \\
\hline FGFR2 & CGD with craniosynostosis & $A D$ & $10 q 26.13$ & 176943 \\
\hline GATA4 & PGD with or without congenital heart defect & $A D$ & $8 p 23.1$ & 600576 \\
\hline MAP3К1 & CGD; PGD & $A D$ & $5 q 11.2$ & 613762 \\
\hline NROB1 & CGD; PGD & $\mathrm{XL}$, duplication & Xp21.2 & 300473 \\
\hline NR5A1 & CGD; PGD; TRS; hypospadias; cryptorchidism & $A R ; A D$ & $9 q 33.3$ & 184757 \\
\hline SOX9 & $\begin{array}{l}\text { CGD or PGD with campomelic and acampomelic } \\
\text { dysplasia }\end{array}$ & $A D$ & $17 q 24.3$ & 608160 \\
\hline SRY & CGD; PGD & YL & Yp11.2 & 480000 \\
\hline TSPYL1 & PGD and sudden infant death & AR & $6 q 22.1$ & 604714 \\
\hline WNT4 & Ovotesticular DSD; CGD (duplication) & $A D$, duplication & $1 \mathrm{p} 36.12$ & 603490 \\
\hline WT1 & $\begin{array}{l}\text { CGD or PGD within Denysh Drash or Frasier } \\
\text { syndrome ; TRS; hypospadias }\end{array}$ & $A D$ & $11 \mathrm{p} 13$ & 607102 \\
\hline WWOX & PGD & $A D$, deletion & $16 q 23.2$ & \\
\hline ZFPM2 & CGD; PGD & $A D$ & $8 q 23.1$ & 603693 \\
\hline \multicolumn{5}{|c|}{ Disorder of androgen synthesis (DAS) } \\
\hline$A K R 1 C 2$ & $3 \alpha$-hydroxysteroid dehydrogenase deficiency & $A R$ & 10p15.1 & 600450 \\
\hline$A K R 1 C 4$ & $3 \alpha$-hydroxysteroid dehydrogenase deficiency & $A R$ & $10 \mathrm{p} 15.1$ & 600451 \\
\hline CYB5A & Cytochrome b5 deficiency & $A R$ & $18 q 22.3$ & 250790 \\
\hline CYP11A1 & P450 side chain cleavage syndrome & $A R$ & $15 q 24.1$ & 118485 \\
\hline CYP17A1 & $\begin{array}{l}\text { Combined 17-hydroxylase, 17,20-lyase } \\
\text { deficiency }\end{array}$ & $A R$ & $10 q 24.32$ & 609300 \\
\hline HSD3B2 & $3 \beta$-hydroxysteroid dehydrogenase II deficiency & $A R$ & $1 \mathrm{p} 12$ & 613890 \\
\hline HSD17B3 & $17 \beta$-hydroxysteroid deficiency type 3 & $A R$ & $9 q 22.32$ & 605573 \\
\hline LHCGR & Leydig cell hypoplasia & $A R$ & $2 \mathrm{p} 16.3$ & 152790 \\
\hline POR & P450 oxidoreductase deficiency & $A R$ & $7 q 11.23$ & 124015 \\
\hline SRD5A2 & $5 \alpha$-reductase deficiency & $A R$ & $2 \mathrm{p} 23.1$ & 607306 \\
\hline STAR & Lipoid congenital adrenal hyperplasia & AR & $8 p 11.23$ & 250790 \\
\hline \multicolumn{5}{|c|}{ Disorder of androgen action (DAA) } \\
\hline$A R$ & CAIS; PAIS; hypospadias & $X L$ & $\mathrm{Xq12}$ & 313700 \\
\hline \multicolumn{5}{|c|}{ Other and syndromic forms of DSD (sDSD) } \\
\hline$A M H$ & PMDS type 1 & $A R$ & $19 p 13.3$ & 600957 \\
\hline AMHR2 & PMDS type 2 & $A R$ & $12 q 13.13$ & 600956 \\
\hline DHCR7 & Smith-Lemli-Opitz syndrome & $A R$ & $11 q 13.4$ & 602858 \\
\hline HOXA13 & Hand-foot-genital syndrome & $A D$ & $7 q 15.2$ & 142959 \\
\hline INSL3 & Cryptorchidism & $A D$ & 19p13.11 & 146738 \\
\hline MAMLD1 & CGD; PGD; hypospadias & $\mathrm{XL}$ & Xq28 & 300120 \\
\hline MID1 & Opitz GBBB syndrome & $\mathrm{XL}$ & Xp22.2 & 300552 \\
\hline RXFP2 & Cryptorchidism & $A D$ & $13 q 13.1$ & 606655 \\
\hline
\end{tabular}

$A R$, autosomal recessive; CAIS, complete androgen insensitivity syndrome; CGD, complete gonadal dysgenesis; PAIS, partial androgen insensitivity syndrome; PGD, partial gonadal dysgenesis; PMDS, persistent Müllerian duct syndrome; TRS, testicular regression syndrome; TRS, testicular regression syndrome; XL, X-linked; YL, Y-linked.

Consistent with the DSD cases in the study, only genotype calls with read depths $>10 \times$ and quality scores $>95$ were used. Additionally, the 37 DSD-related genes were covered in both the whole exome data of the CoLaus cohort and the TruSight One Sequencing Panel Kit of the DSD cohort to an average read depth of $>20 \times$ for $>99 \%$ of the nucleotides in the genes.

$\begin{array}{lr}\text { https://ec.bioscientifica.com } & \text { ○ } 2018 \text { The authors } \\ \text { https://doi.org/10.1530/EC-18-0472 } & \text { Published by Bioscientifica Ltd }\end{array}$

Case vs control statistical analysis for an enrichment of individual variants or oligogenicity was performed using a two-tailed Fisher's exact test. The Mann-Whitney $U$ test was performed to assess the difference in EMS in cases reaching the definitive diagnosis vs cases with no final diagnosis. A statistic of $P<0.05$ was considered to be significant.

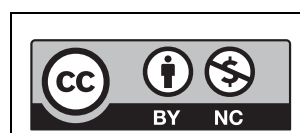

This work is licensed under a Creative Commons Attribution-NonCommercial 4.0 International License. 


\section{Results}

\section{Clinical approach based on physical and endocrine evaluation}

After structured clinical assessment followed by thorough endocrine evaluation including biochemical and radiological tests, each case was attributed a clinical diagnosis. All data regarding phenotyping are provided in Supplementary Tables 3 and 4. The median EMS was 4.5 (0-9). In all cases with low plasma level of testosterone (basal value if assessed during expected minipuberty/ puberty or after hCG test if assessed during childhood) and $\mathrm{AMH}$, a subcategory of DGD was attributed. Among this subgroup, there were (i) five patients with testicular regression syndrome (TRS), as despite lack of testicles they presented with male external genitalia and no Müllerian structures were identified on the ultrasound and (ii) four patients with complete gonadal dysgenesis (CGD), as they presented with female external genitalia, high plasma gonadotropins and an infantile uterus. Additionally, for patients with low plasma level of testosterone or high testosterone/DHT ratio, an AMH serum level within normal ranges, and no Müllerian structures, a subcategory of DAS (disorder of androgen synthesis) was established. Namely, there were three patients suspected for a $5 \alpha$-reductase deficiency $(5 \alpha \mathrm{RD})$ and one patient with undetectable testosterone plasma level and no uterus on the ultrasound indicating proper level of AMH during fetal life. In addition, a subcategory of disorder of androgen action (DAA) was attributed to those with proper gonadal function reflected by normal to high plasma levels of testosterone and AMH and no Müllerian structures. Namely, six patients with atypical genitalia received a phenotypic diagnosis of NSDUM, and eight patients were diagnosed with either a complete or partial form of androgen insensitivity syndrome (CAIS or PAIS) depending on the appearance of the external genitalia. Lastly, there were eight children that received a phenotypic diagnosis of syndromic DSD, as they presented with multiple associated conditions (i.e.: psychomotor delay, dysmorphic features, congenital heart defect). Additionally, although the majority of children presented with normal adrenal function, there were two syndromic patients with a primary (ID 32) and a secondary adrenal insufficiency (ID 30). (Fig. 1, Supplementary Tables 3 and 4).

\section{Array-CGH}

Given the incidence of genomic rearrangements in patients with DGD and syndromic forms of DSD, array-CGH was first performed on 10 of the 17 patients with CGD, TRS or sDSD (Fig. 2). No pathogenic genomic structural abnormalities were identified in these patients.

\section{Sequencing results and their relationship to clinical diagnosis}

Sequencing of the 37 genes implicated in DSD was performed successfully in all 35 patients (Fig. 2). The results were filtered according to the strategy described in the methods, including ACMG variant classification. This analysis identified 11 pathogenic variants, 2 likely pathogenic variants and 17 VUS (Table 2). Interestingly, each patient harbored at least one variant in 15 out of the 37 DSD-related genes, and most frequently, these were missense mutations. There were recurrent mutations in the $A R$ and $H S D 17 B 3$ genes in unrelated patients (Table 2).

As a definitive genetic diagnosis was considered when pathogenic or likely pathogenic variants were identified, 12 cases were assigned a definitive genetic diagnosis. In the remaining patients, the final diagnosis remained at the clinical level, as either no variant was identified $(n=15)$ or at least one VUS was found $(n=8)$ (Fig. 3). In the majority of cases, the variant was classified as VUS primarily due to a discordance between the phenotype and genetic mutation or, in cases with expansion of the number of CAG repeats, due to undetermined significance of its contribution to the DSD phenotype. Overall, 13 patients were found to harbor at least one VUS.

Among those with a genetically confirmed diagnosis $(n=12)$, nine patients had a clinical and endocrine evaluation that enabled an accurate initial identification of the underlying genetic cause. Namely, in seven patients suspected for CAIS and one for NSDUM, the pathogenic variant was identified in the $A R$ gene, as well as one case classified with DAS was found to harbor compound heterozygote variants in the HSD17B3 gene. However, in three patients, the result of the genetic assessment led to the re-evaluation of the initial clinical diagnosis which subsequently led to diagnostic reclassification. Specifically, in patients suspected for a disorder of androgen action (ID 14 and 27), variants in the WT1 and NR5A1 genes, respectively, were identified indicating a disorder of gonadal dysgenesis. Additionally, a patient initially classified with $5 \alpha$-reductase deficiency (ID 11) was found to harbor a likely pathogenic variant in the HOXA13 gene implicated in syndromic forms of DSD.

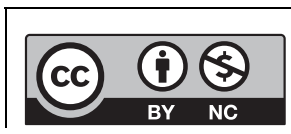

This work is licensed under a Creative Commons Attribution-NonCommercial 4.0 International License. 


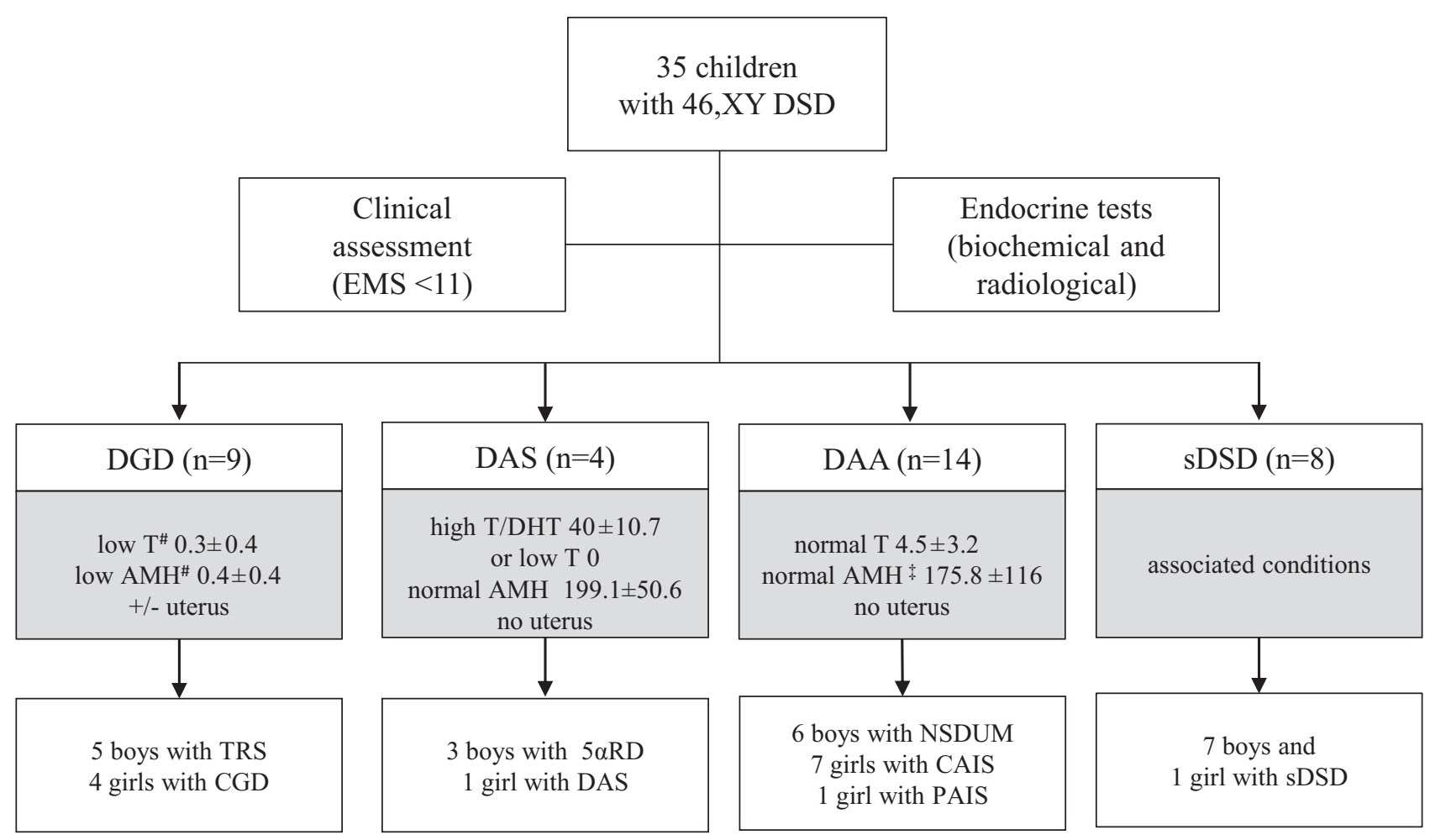

\section{Figure 1}

Identified phenotypes based on clinical assessment and endocrine tests. AMH, anti-Müllerian hormone; CAIS, complete androgen insensitivity syndrome CGD, complete gonadal dysgenesis; $5 \alpha$-reductase deficiency; DAA, disorder of androgen action; DAS, disorder of androgen synthesis; DGD, disorder of gonadal development; DHT, dihydrotestosterone; DSD, disorder of sex development; EMS, external masculinization score; NSDUM, non-syndromic disorder of undermasculinization; PAIS, partial androgen insensitivity syndrome; SDSD, syndromic DSD; TRS, testicular regression syndrome; testosterone levels, if not assessed during minipuberty or puberty, were after hCG stimulation test if available; ${ }^{*}(\mathrm{ng} / \mathrm{mL}){ }^{\dagger}{ }^{\dagger}$ for pubertal boys $(n=2)$ inhibin B level was considered.

\section{Factors correlating with achieving a definitive genetic diagnosis}

Patients for whom a definitive genetic diagnosis was rendered had a significantly lower mean EMS score (i.e. had a more severe phenotype), than patients with either a VUS or with no variant (2.5 vs $6, P=0.04)$. Additionally, the highest genetic diagnostic yield was seen in cases initially suspected of DAA (10 patients out of 14), followed by patients classified as DAS (two patients out of four). However, a final genetic diagnosis was not possible in any of the patients suspected of DGD or sDSD.

\section{Oligogenic inheritance in DSD patients}

There were eight patients (23\%) harboring a mutation in more than one DSD gene (Table 2). To evaluate whether the DSD cohort was statistically enriched for oligogenicity, we compared the results to exome sequencing data from the 247 male participants in the 'Cohorte Lausannoise' CoLaus control population. As ACMG criteria are not intended for use in non-monogenic disorders (20), variants in
37 DSD-related genes were filtered for non-synonymous variants with a MAF $<1.0 \%$, including nonsense, splicesite ( \pm 6 base pairs) and missense variants found to be damaging in at least one of the two protein prediction programs, SIFT or MutationTaster. There was a statistical enrichment in oligogenic variants in our DSD cohort compared to CoLaus controls ( $23 \%$ vs $2.5 \% ; P=0.0003)$.

\section{Variants in known $\mathrm{CHH}$ genes}

Despite the fact that DSD patients typically do not present with hypogonadotropic hypogonadism, there is a small overlap in clinical presentation, namely the presence of micropenis and/or cryptorchidism. Therefore, several groups have previously explored a potential genetic overlap in these patients related to the known $\mathrm{CHH}$ genes $(6,7)$. Similarly, we evaluated our DSD cohort, within which four heterozygous and one hemizygous rare variants in the known $\mathrm{CHH}$ genes were identified in five DSD patients (14\%) who actually presented with high gonadotrophin plasma level (Supplementary Tables 4 and 5). There was no statistically significant difference

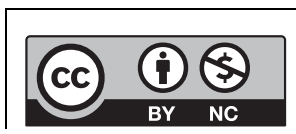

This work is licensed under a Creative Commons Attribution-NonCommercial 4.0 International License. 


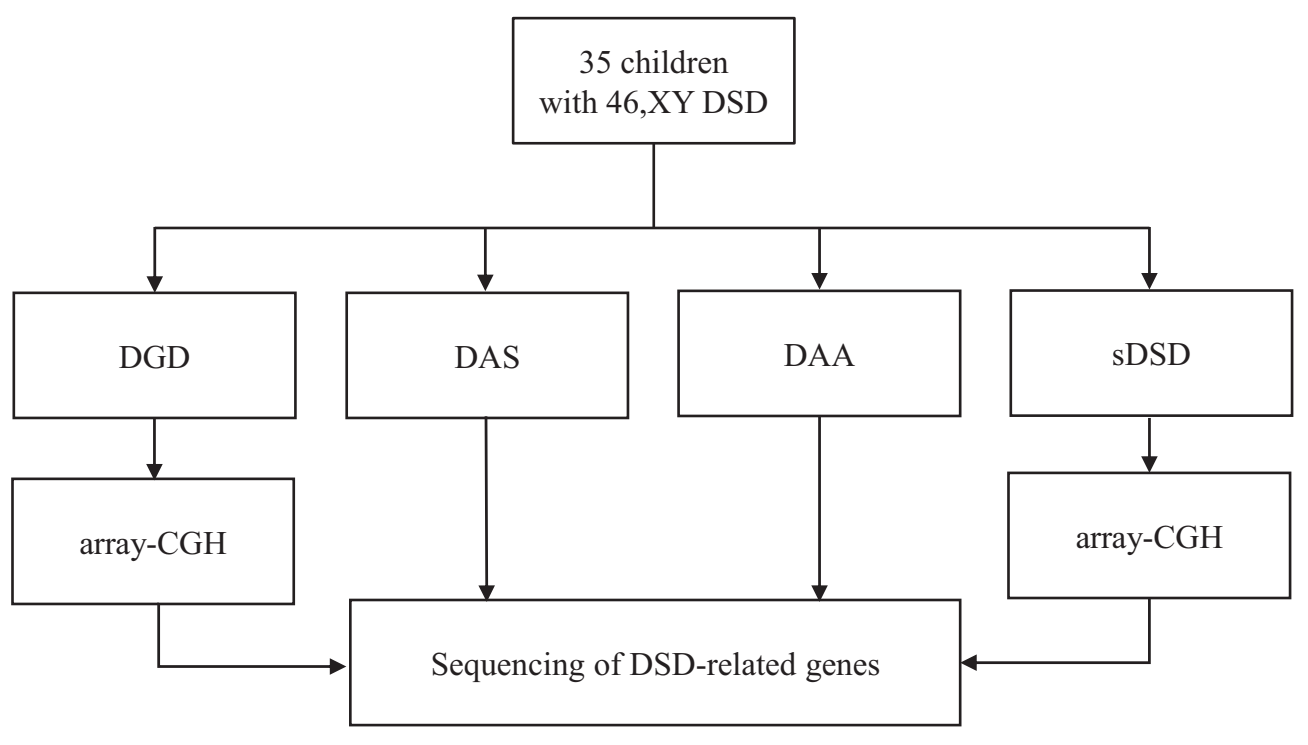

\section{Figure 2}

Genetic approach according to different DSD subcategories. array-CGH, array comparative genomic hybridization; DAA, disorder of androgen action; DAS, disorder of androgen synthesis; DGD, disorder of gonadal development; DSD, disorder of sex development; sDSD, syndromic DSD. between the DSD cohort and the CoLaus controls (14\% vs $20 \%, P=0.3$ ) at the individual variant level, gene level or overall panel of genes.

\section{Discussion}

Despite the introduction of NGS methods into the clinical setting, the elucidation of genetic background in $46, \mathrm{XY}$ DSD remains challenging. We presented the results on the integrated approach combining clinical and genetic evaluation of a cohort of $46, \mathrm{XY}$ DSD patients systematically assessed in one center. This integrated approach resulted in identification of genetic findings in one-third of patients, while the final diagnosis in rest of them remained clinical only. As expected, a wide spectrum of phenotypes was observed leading to further subcategorization of the DSD cohort into DGD, DAS, DAA and sDSD. Thus, although the sample size was limited, the cohort appeared to be representative.

Importantly, the NGS of 37 DSD-related genes produced valuable results. The diagnostic yield (i.e. percentage of samples with pathogenic or likely pathogenic genetic results) in our cohort was 34\% (12/35), which is comparable to other reports $(24,25)$. In the majority of patients with a definitive diagnosis (9/12), physical and endocrine evaluation was sensitive and/or specific enough to clearly identify the underlying genetic cause. However, with the exception of one case classified as DAS, this was only true for patients suspected of DAA - a condition with the strongest correlation between phenotype and genotype when presenting with a CAIS phenotype (2).
Given the variable specificity of endocrine tests to accurately diagnose a phenotype (i.e. androgens' ratios in the diagnosis of DAS $(11,26,27))$ and since some conditions may present with a wide spectrum of phenotypes (i.e. phenotypes associated with genes such as NR5A1 (28) or WT1 (29)), an accurate diagnosis may only be possible with parallel genetic evaluation. Interestingly, the genetic results obtained in the present study lead to the re-evaluation of the clinical diagnosis in 3 patients, and the subsequent reclassification of their diagnosis. Specifically, one patient (ID 11) with distal limb anomalies, an abnormal testosterone/DHT ratio and initially suspected of $5 \alpha \mathrm{RD}$ was finally accurately diagnosed with a hand-foot-genital syndrome after the identification of a pathogenic variant in the HOXA13 gene (30). Of note, a clinical diagnosis of suspected $5 \alpha \mathrm{RD}$ was not genetically confirmed in any of the patients in our cohort, further confirming the limited utility of testosterone/DHT ratios in the diagnosis of $5 \alpha$-reductase deficiency. Furthermore, two patients (ID 14 and 27) were initially suspected as having DAA, but were found to harbor likely pathogenic mutations in the WT1 and NR5A1 genes, respectively. It has been established that mutations in both genes are implicated in a broad spectrum of phenotypes. In the case of mutations in the WT1 gene, phenotypes can range from hypospadias $(29,31)$ to much more severe phenotypes such as WAGR (Wilms tumor, aniridia, genitourinary anomalies, and mental retardation), Frasier or DenysDrash syndromes $(1,29)$. Phenotypes for patients carrying mutations in the NR5A1 gene cover a spectrum of different degrees of gonadal dysgenesis, with or without associated adrenal insufficiency (28).
This work is licensed under a Creative Commons Attribution-NonCommercial 4.0 International License. 


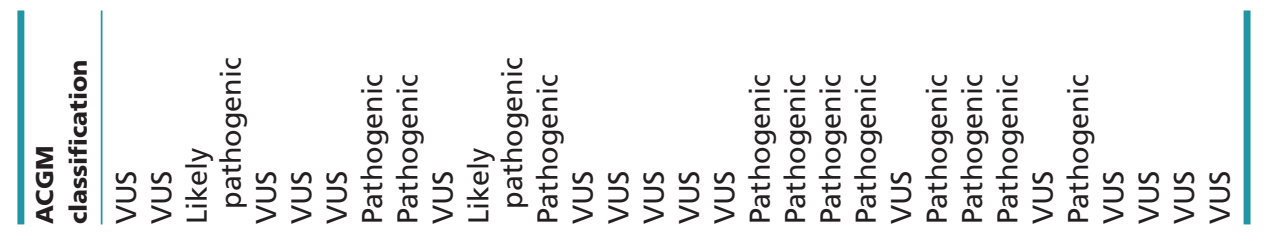

ड़े

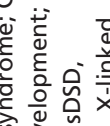

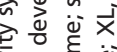

攴口 0 ค

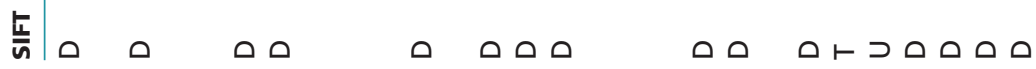

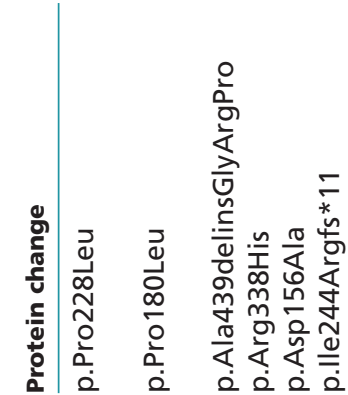
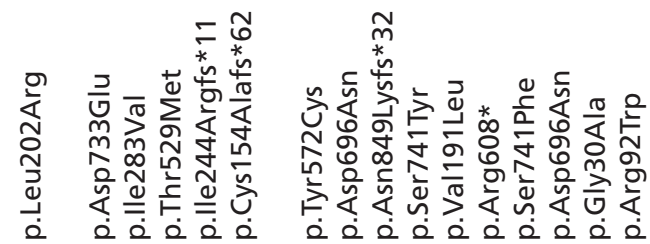

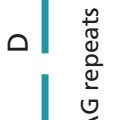

츤 $\times$

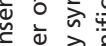

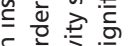

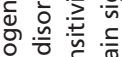

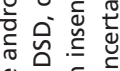

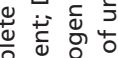

हो

s)

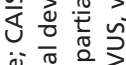

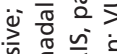

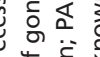

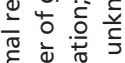

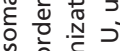

帘

过 हैं

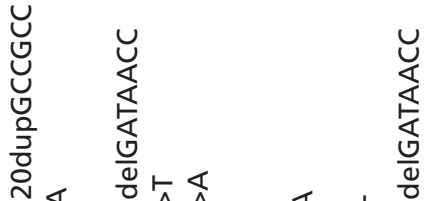

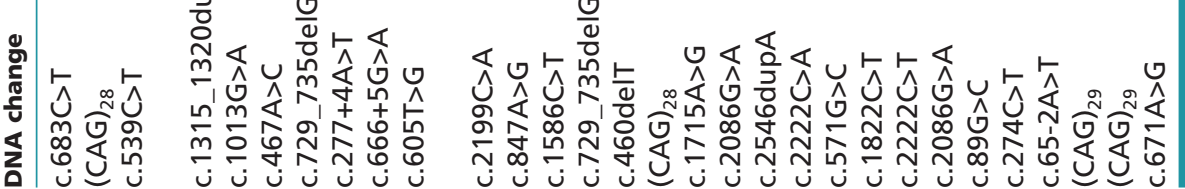

范然

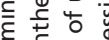

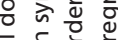

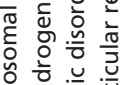

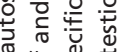

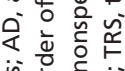

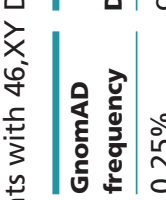

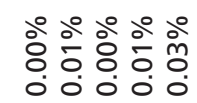

送

$\stackrel{\circ}{\circ} \stackrel{\circ}{\circ}$

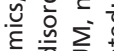

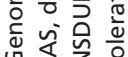

它究离

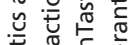

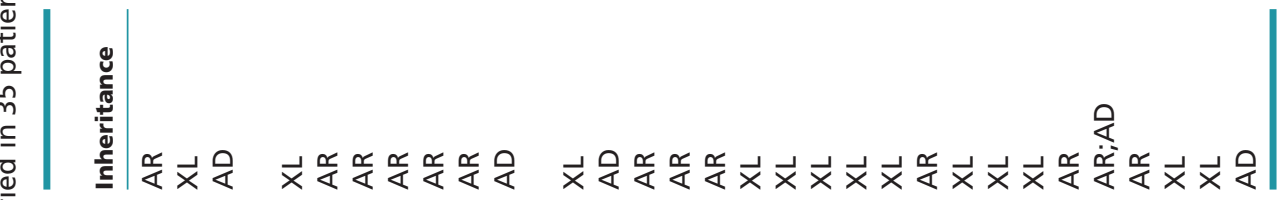

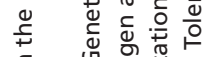

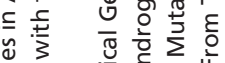

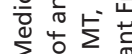

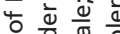

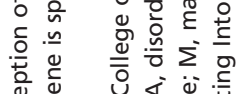

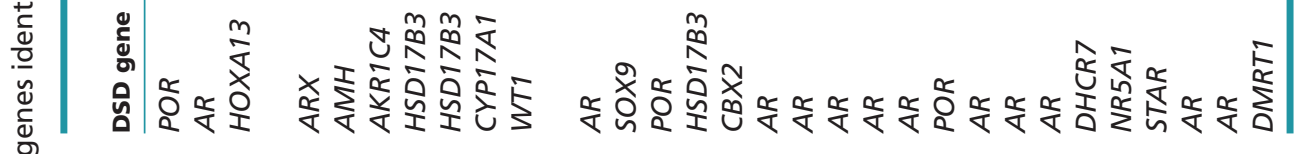

厄)

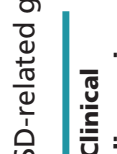

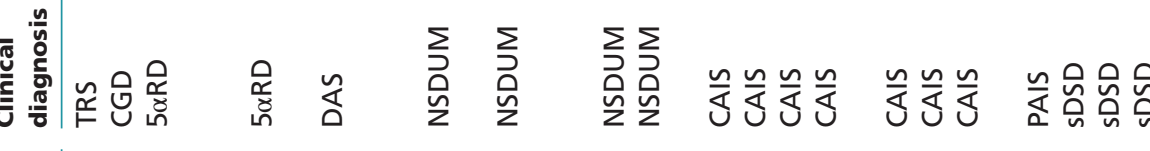

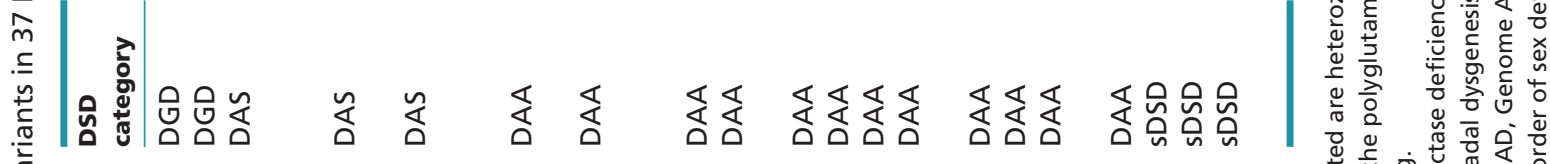

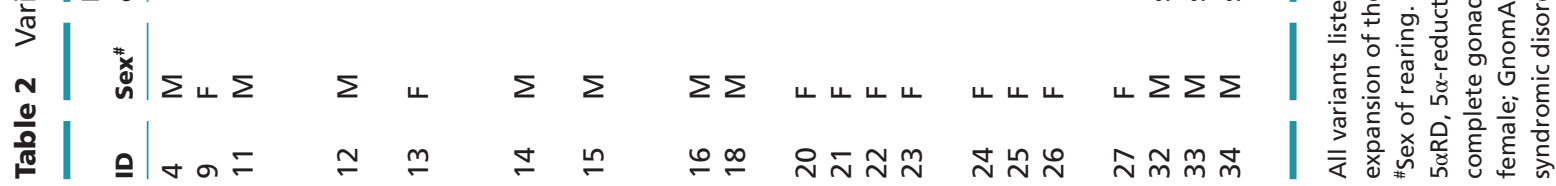




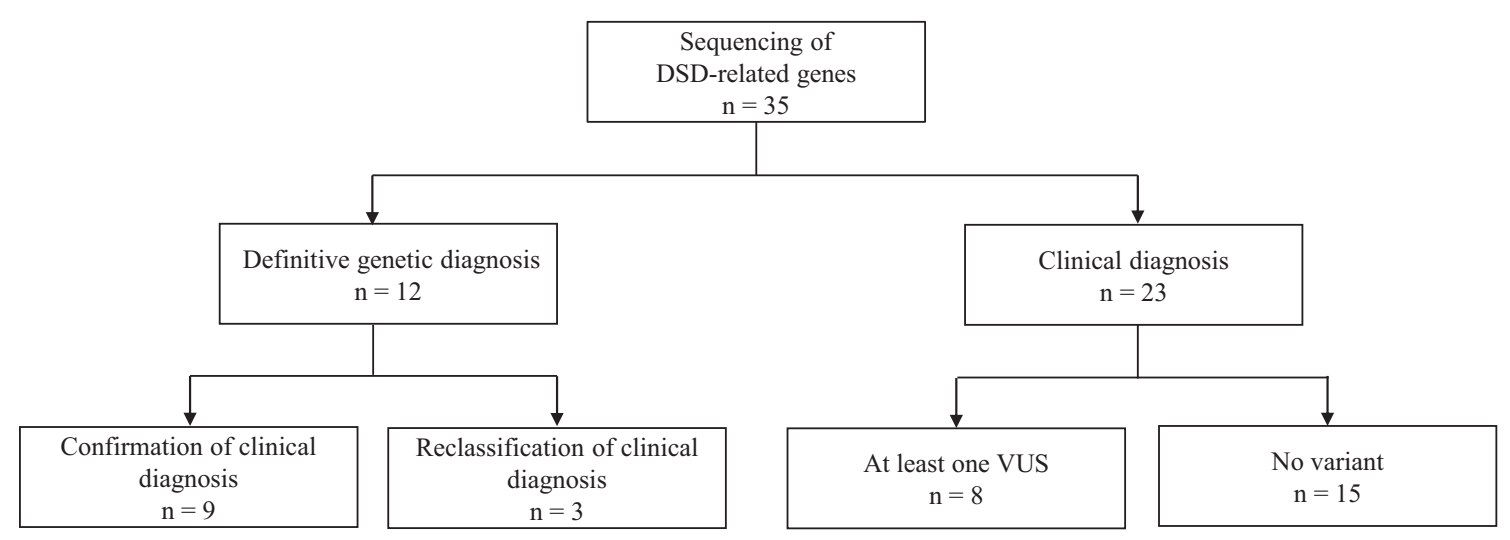

Figure 3

Integrated approach in 46,XY DSD patients. DSD, disorders of sex development; VUS, variant of uncertain significance.

Our study showed that patients with a more severe phenotype (mean EMS score 2.5) were more likely to have a conclusive genetic diagnosis than those with less severe phenotypes (mean EMS score 6), $P=0.04$. This lower diagnostic yield in children with a less severe phenotype is likely due to several reasons. Since it is difficult to definitively draw a line between the DSD and the mild and common urogenital defect such as hypospadias (14) that can be caused by environmental factors as well as genetic polymorphisms $(31,32)$, the enrollment of cases with signs of undermasculinization into the study group may have resulted in a bias. However, as it is known that the degree of undermasculinization reflected by lower EMS does not always increase the likelihood of a diagnostic yield $(5,33)$, it is debatable to exclude mild DSD cases from systematic assessment.

The diagnostic rate differed among DSD subcategories. The highest diagnostic rate (10 cases out of 14) was seen among suspected DAA patients, followed by the DAS subgroup (two cases out of four). Despite the small sample size, these findings are consistent with the results of a targeted gene study on the largest DSD cohort to date (6). However, in contrast with other report, no definitive genetic diagnosis was able to be rendered in patients with syndromic DSD or DGD. One of the reasons might have been the inclusion criteria, as only those patients with CGD who were 'SRY negative' prior to the study were enrolled. Since the $S R Y$ gene constitutes the primary candidate gene for this disorder (1), the likelihood of identifying the genetic etiology of gonadal dysgenesis among 'SRY negative' cases is lower. Furthermore, the subgroup with DGD was represented by cases with TRS, a condition in which environmental factors also play a role (34). Thus, although in some patients the genetic causes were identified in TRS $(35,36)$, the possible presence of

https://ec.bioscientifica.com
https://doi.org/10.1530/EC-18-0472

non-genetic effects lowers the odds of a genetic diagnosis. In addition, although the published incidence of genomic rearrangements in DGD or SDSD patients varies between 20 and $30 \%(3,4,5,11)$, our current study did not identify any pathogenic $\mathrm{CNV}$ in these patients. These negative array-CGH results are likely due the limited number of cases assessed $(n=10)$. Finally, the full spectrum of the genetic determinants of DGD and SDSD is yet to be elucidated; thus, a genetic diagnosis can only be given for a minority of cases (1).

The sequencing of 37 DSD-related genes led to the identification of eight patients (23\%) exhibiting oligogenic inheritance of more than one mutation in DSD genes, and this was not observed in controls $(2.5 \% ; P=0.0003)$. This finding further reinforces recent studies demonstrating that oligogenicity is present in DSD patients and may at least partly explain the incomplete penetrance and variable expressivity within and across DSD families (6, $7,8)$. It is important to note, however, that our current study evaluated oligogenicity in only DSD genes, and did not include variants in known $\mathrm{CHH}$ genes. The fact that this strong association is present even when not including $\mathrm{CHH}$ genes (as previous studies have done (6, 7)), coupled with a statistically equal number of $\mathrm{CHH}$ variants in both our DSD cohort and the CoLaus control population suggests a minimal or at best modifying role of $\mathrm{CHH}$ genes in the pathogenesis of DSD. Further, the role of $\mathrm{CHH}$ genes in DSD is debatable, given their distinct pathogenesis and endocrine profiles, as well as our results showing a nearly identical frequency of rare $\mathrm{CHH}$ variants in both DSD patients and CoLaus controls. However, larger genetic studies coupled with in vivo and/or in vitro functional studies will help to further clarify this.

In summary, the study clearly showed that an integrated approach is the best routine practice in the 
diagnosis of $46, \mathrm{XY}$ DSD. In those cases with a wide phenotype variability and reduced utility of conventional endocrine tests to fully determine the phenotype, reaching the definitive diagnosis may only be possible with parallel genetic testing. On the other hand, substantial prevalence of variants of uncertain significance or of possible oligogenic nature with different contribution to the phenotype underlay the importance of thorough clinical assessment. Therefore, in order to overcome potential obstacles in rendering an accurate diagnosis, data from clinical, biochemical and genetic assessment should be analyzed in an integrative manner. This approach should be then followed by reporting the data and comparing with other centers. Furthermore, only one third of patients harbor pathogenic mutations in DSD genes. The remaining patients argue for multi-national studies to identify additional genes involved in the pathogenesis of DSD.

\section{Supplementary data}

This is linked to the online version of the paper at https://doi.org/10.1530/ EC-18-0472.

\section{Declaration of interest}

The authors declare that there is no conflict of interest that could be perceived as prejudicing the impartiality of the research reported.

\section{Funding}

This study was supported by the Polish National Science Centre grant (2014/12/N/NZ5/00448). The array-CGH analysis was supported by Poznan University of Medical Science grant (502-14-01126186-09772).

\section{References}

1 Ahmed SF, Bashamboo A, Lucas-Herald A \& McElreavey K. Understanding the genetic aetiology in patients with XY DSD. British Medical Bulletin 2013106 67-89. (https://doi.org/10.1093/bmb/ldt008)

2 Ahmed SF, Cheng A, Dovey L, Hawkins JR, Rowland J, Shimura N, Tait AD \& Hughes IA. Phenotypic features, androgen receptor binding, and mutational analysis in 278 clinical cases reported as androgen insensitivity syndrome. Journal of Clinical Endocrinology and Metabolism 200085 658-665. (https://doi.org/10.1210/ jcem.85.2.6337)

3 Ledig S, Hiort O, Scherer G, Hoffmann M, Wolff G, Morlot S, Kuechler A \& Wieacker P. Array-CGH analysis in patients with syndromic and non-syndromic XY gonadal dysgenesis: evaluation of array CGH as diagnostic tool and search for new candidate loci. Human Reproduction 201010 2637-2646. (https://doi.org/10.1093/ humrep/deq167)

4 White S, Ohnesorg T, Notini A, Roeszler K, Hewitt J, Daggag H, Smith C, Turbitt E, Gustin S, van den Bergen J, et al. Copy number variation in patients with disorders of sex development due to 46,XY gonadal dysgenesis. PLoS ONE 20116 e17793. (https://doi. org/10.1371/journal.pone.0017793)
5 Nixon R, Cerqueira V, Kyriakou A, Lucas-Herald A, McNeilly J, McMillan M, Purvis AI, Tobias ES, McGowan R \& Ahmed SF. Prevalence of endocrine and genetic abnormalities in boys evaluated systematically for a disorder of sex development. Human Reproduction 201732 2130-2137. (https://doi.org/10.1093/humrep/dex280)

6 Eggers S, Sadedin S, van den Bergen JA, Robevska G, Ohnesorg T, Hewitt J, Lambeth L, Bouty A, Knarston IM, Tan TY, et al. Disorders of sex development: insights from targeted gene sequencing of a large international patient cohort. Genome Biology 201617243. (https://doi.org/10.1186/s13059-016-1105-y)

7 Wang H, Zhang L, Wang N, Zhu H, Han B, Sun F, Yao H, Zhang Q, Zhu W, Cheng T, et al. Next-generation sequencing reveals genetic landscape in 46,XY disorders of sexual development patients with variable phenotypes. Human Genetics 201837 265-277. (https://doi. org/10.1007/s00439-018-1879-y)

8 Camats N, Fernández-Cancio M, Audí L, Schaller A \& Flück CE. Broad phenotypes in heterozygous NR5A1 46,XY patients with a disorder of sex development: an oligogenic origin? European Journal of Human Genetics 201826 1329-1338. (https://doi.org/10.1038/s41431-0180202-7)

9 Baxter RM, Arboleda VA, Lee H, Barseghyan H, Adam MP, Fechner PY, Bargman R, Keegan C, Travers S, Schelley S, et al. Exome Sequencing for the Diagnosis of 46,XY Disorders of Sex Development. Journal of Clinical Endocrinology and Metabolism 2015100 333-344. (https://doi. org/10.1210/jc.2014-2605)

10 Audí L, Ahmed SF, Krone N, Cools M, McElreavey K, Holterhus PM, Bashamboo A, Hiort O, Wudy SA \& McGowanR. Genetics in Endocrinology: Approaches to molecular genetic diagnosis in the management of differences/disorders of sex development (DSD): position paper of EU COST Action BM 1303 "DSDnet". European Journal of Endocrinology 201818 0256. (https://doi.org/10.1530/EJE18-0256)

11 Alhomaidah D, McGowan R \& Ahmed SF. The current state of diagnostic genetics for conditions affecting sex development. Clinical Genetics 201791 157-162. (https://doi.org/10.1111/cge.12912)

12 Ahmed SF, Khwaja O \& Hughes IA. The role of a clinical score in the assessment of ambiguous genitalia. BJU International $2000 \mathbf{8 5}$ 120-124. (https://doi.org/10.1046/j.1464-410x.2000.00354.x)

13 Ahmed SF, Achermann JC, Arlt W, Balen A, Conway G, Edwards Z, Elford S, Hughes IA, Izatt L, Krone N, et al. Society for Endocrinology UK guidance on the initial evaluation of an infant or an adolescent with a suspected disorder of sex development (Revised 2015). Clinical Endocrinology 201684 771-788. (https://doi.org/10.1111/cen.12857)

14 Ahmed SF \& Rodie M. Investigation and initial management of ambiguous genitalia. Best Practice and Research. Clinical Endocrinology and Metabolism 201024 197-218. (https://doi.org/10.1016/j. beem.2009.12.001)

15 Ahmed SF, Keir L, McNeilly J, Galloway P, O’Toole S \& Wallace AM. The concordance between serum anti-Mullerian hormone and testosterone concentrations depends on duration of hCG stimulation in boys undergoing investigation of gonadal function. Clinical Endocrinology 201072 814-819. (https://doi.org/10.1111/j.13652265.2009.03724.x)

16 Pang S, Levine LS, Chow D, Sagiani F, Saenger P \& New MI. Dihydrotestosterone and its relationship to testosterone in infancy and childhood. Journal of Clinical Endocrinology and Metabolism 1979 48 821-826. (https://doi.org/10.1210/jcem-48-5-821)

17 Alikaşifoğlu A, Vurallı D, Hiort O, Gönç N, Özön A \& Kandemir N. Severe Undervirilisation in a 46 ,XY Case Due to a Novel Mutation in HSD17B3 Gene. Journal of Clinical Research in Pediatric Endocrinology 20157 249-252. (https://doi.org/10.4274/jcrpe.2069)

18 MacArthur DG, Manolio TA, Dimmock DP, Rehm HL, Shendure J, Abecasis GR, Adams DR, Altman RB, Antonarakis SE, Ashley EA, et al. Guidelines for investigating causality of sequence variants in human disease. Nature 2014508 469-476. (https://doi.org/10.1038/ nature13127)

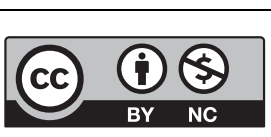

This work is licensed under a Creative Commons Attribution-NonCommercial 4.0 International License. 
19 Beck TF, Mullikin JC, NISC Comparative Sequencing Program \& Biesecker LG. Systematic Evaluation of Sanger Validation of NextGeneration Sequencing Variants. Clinical Chemistry 201662 647-654. (https://doi.org/10.1373/clinchem.2015.249623)

20 Richards S, Aziz N, Bale S, Bick D, Das S, Gastier-Foster J, Grody WW, Hegde M, Lyon E, Spector E, et al. Standards and guidelines for the interpretation of sequence variants: a joint consensus recommendation of the American College of Medical Genetics and Genomics and the Association for Molecular Pathology. Genetics in Medicine 201517 405-424. (https://doi.org/10.1038/gim.2015.30)

21 Lim HN, Chen H, McBride S, Dunning AM, Nixon RM, Hughes IA \& Hawkins JR. Longer polyglutamine tracts in the androgen receptor are associated with moderate to severe undermasculinized genitalia in XY males. Human Molecular Genetics 20009 829-834. (https://doi. $\operatorname{org} / 10.1093 / \mathrm{hmg} / 9.5 .829)$

22 Aschim EL, Nordenskjöld A, Giwercman A, Lundin KB, Ruhayel Y, Haugen TB, Grotmol T \& Giwercman YL. Linkage between cryptorchidism, hypospadias, and GGN repeat length in the androgen receptor gene. Journal of Clinical Endocrinology and Metabolism 200489 5105-5109. (https://doi.org/10.1210/ jc.2004-0293)

23 Firmann M, Mayor V, Vidal PM, Bochud M, Pecoud A, Hayoz D, Paccaud F, Preisig M, Song KS, Yuan X, et al. The CoLaus study: a population-based study to investigate the epidemiology and genetic determinants of cardiovascular risk factors and metabolic syndrome. BMC Cardiovascular Disorders 20088 6. (https://doi. org/10.1186/1471-2261-8-6)

24 Arboleda VA, Lee H, Sánchez FJ, Sandberg DE, Grody WW, Nelson SF \& Vilain E. Targeted massively parallel sequencing provides comprehensive genetic diagnosis for patients with disorders of sex development. Clinical Genetics 201383 35-43. (https://doi. org/10.1111/j.1399-0004.2012.01879.x)

25 Kim JH, Kang E, Heo SH, Kim GH, Jang JH, Cho EH, Lee BH, Yoo HW $\&$ Choi JH. Diagnostic yield of targeted gene panel sequencing to identify the genetic etiology of disorders of sex development. Molecular and Cellular Endocrinology 2017444 19-25. (https://doi. org/10.1016/j.mce.2017.01.037)

26 Maimoun L, Philibert P, Cammas B, Audran F, Bouchard P, Fenichel P, Cartigny M, Pienkowski C, Polak M, Skordis N, et al. Phenotypical, biological, and molecular heterogeneity of $5 \alpha$-reductase deficiency: an extensive international experience of 55 patients. Journal of Clinical Endocrinology and Metabolism 201196 296-307. (https://doi. org/10.1210/jc.2010-1024)
27 Ahmed SF, Iqbal A \& Hughes IA. The testosterone:androstenedione ratio in male undermasculinization. Clinical Endocrinology 200053 697-702. (https://doi.org/10.1046/j.1365-2265.2000.01166.x)

28 Domenice S, Machado AZ, Ferreira FM, Ferraz-de-Souza B, Lerario AM, Lin L, Nishi MY, Gomes NL, da Silva TE, Silva RB, et al. Wide spectrum of NR5A1-related phenotypes in 46,XY and 46,XX individuals. Birth Defects Research. Part C, Embryo Today: Reviews 2016 108 309-332. (https://doi.org/10.1002/bdrc.21145)

29 Köhler B, Biebermann H, Friedsam V, Gellermann J, Maier RF, Pohl M, Wieacker P, Hiort O, Grüters A \& Krude H. Analysis of the Wilms' tumor suppressor gene (WT1) in patients 46,XY disorders of sex development. Journal of Clinical Endocrinology and Metabolism 201196 E1131-E1136. (https://doi.org/10.1210/jc.2010-2804)

30 Goodman FR, Bacchelli C, Brady AF, Brueton LA, Fryns JP, Mortlock DP, Innis JW, Holmes LB, Donnenfeld AE, Feingold M, et al. Novel HOXA13 mutations and the phenotypic spectrum of handfoot-genital syndrome. American Journal of Human Genetics 200067 197-202. (https://doi.org/10.1086/302961)

31 Kalfa N, Gaspari L, Ollivier M, Philibert P, Bergougnoux A, Paris F \& Sultan C. Molecular genetics of hypospadias and cryptorchidism recent developments. Clinical Genetics 201895 122-131. (https://doi. org/10.1111/cge.13432)

32 van der Zanden LF, van Rooij IA, Feitz WF, Franke B, Knoers NV \& Roeleveld N. Aetiology of hypospadias: a systematic review of genes and environment. Human Reproduction Update 201218 260-283. (https://doi.org/10.1093/humupd/dms002)

33 Baetens D, Mladenov W, Delle Chiaie B, Menten B, Desloovere A, Iotova V, Callewaert B, Van Laecke E, Hoebeke P, De Baere E, et al. Extensive clinical, hormonal and genetic screening in a large consecutive series of $46, \mathrm{XY}$ neonates and infants with atypical sexual development Orphanet Journal of Rare Diseases. 20149 209. (https:// doi.org/10.1186/s13023-014-0209-2)

34 Bar-Maor JA, Groisman G \& Lam M. Antenatal torsion of the testes, a cause of vanishing testis syndrome. Pediatric Surgery International 19938 236-238. (https://doi.org/10.1007/BF00182526)

35 Philibert P, Zenaty D, Lin L, Soskin S, Audran F, Léger J, Achermann JC \& Sultan C. Mutational analysis of steroidogenic factor 1 (NR5a1) in 24 boys with bilateral anorchia: a French collaborative study. Human Reproduction 200722 3255-3261. (https://doi.org/10.1093/humrep/dem278)

36 McElreavey K \& Fellous M. Sex determination and the Y chromosome. American Journal of Medical Genetics 199989 176-185. (https://doi.org/10.1002/(SICI)1096-8628(19991229)89:4)

Received in final form 8 November 2018

Accepted 28 November 2018

Accepted Preprint published online 28 November 2018

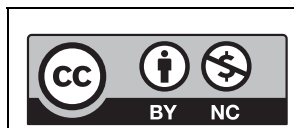

This work is licensed under a Creative Commons Attribution-NonCommercial 4.0 International License. 